\title{
Promoting Crystal Distribution Uniformity Based on the CVD Method with the Aid of Finite Element
}

Methods

Feng Zhang, ${ }^{a}$ Zeping Wang, ${ }^{a}$ Kai Zheng, ${ }^{a}$ Caining Feng, ${ }^{b}$ Ran Yao, ${ }^{c}$ Luqi Tao, ${ }^{c}$ Jiabing Yu, ${ }^{a}$ and Xianping Chen ${ }^{*}, a, c$

${ }^{a}$ Key Laboratory of Optoelectronic Technology \& Systems, Education Ministry of China, Chongqing University, Chongqing 400044, China

${ }^{\mathrm{b}}$ The Faculty of Mechanical and Electrical Engineering, Guilin University of Electronic Technology, Guilin 541004, China

${ }^{c}$ The State Key Laboratory of Power Transmission Equipment and System Security and New Technology, Chongqing University, Chongqing 400044, China 
Table S1. Detailed parameters used in simulation and experiment

\begin{tabular}{|c|c|}
\hline Variable name & Value (mm) \\
\hline Length of quartz boat & 100 \\
\hline outer diameter of quartz boat & 15 \\
\hline The thickness of quartz boat wall & 1 \\
\hline Length of silica/silicon substrate & 20 \\
\hline Width of silica/silicon substrate & 15 \\
\hline The thickness of silica/silicon substrate & 1 \\
\hline Outer diameter of quartz tube & 40 \\
\hline The thickness of quartz tube & 3.5 \\
\hline
\end{tabular}

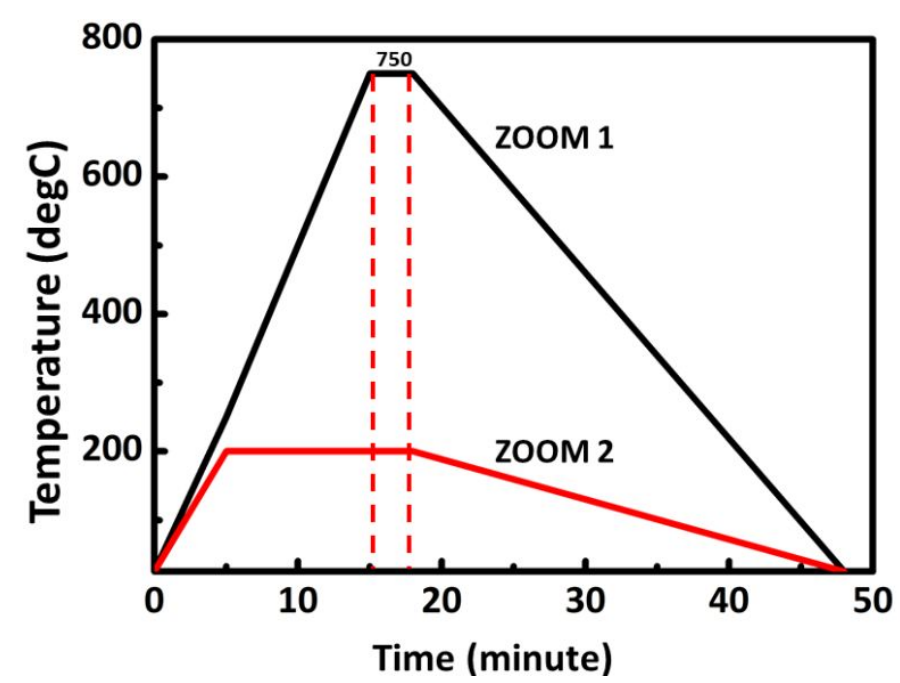

Figure S1. The heating process in two heating zoom.

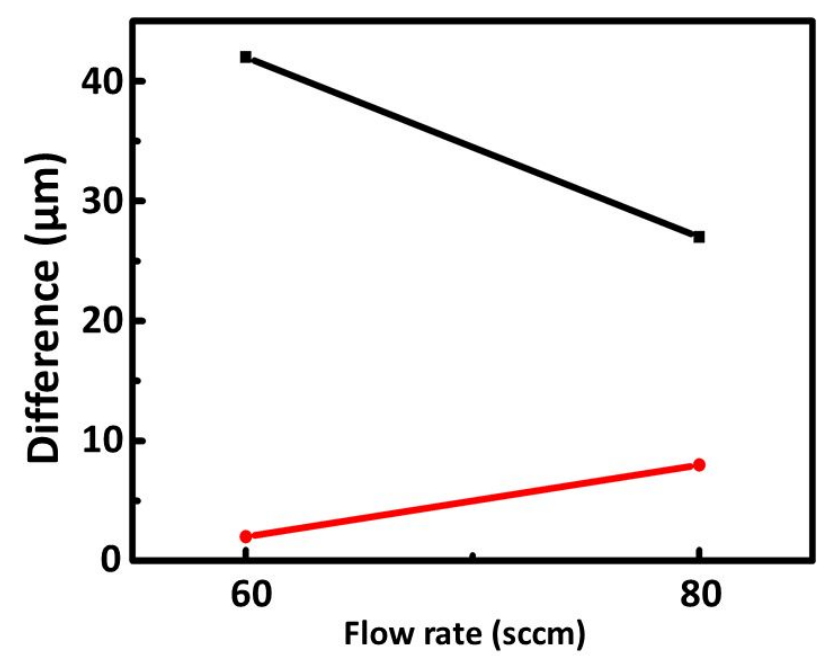

Figure S2. The maximum crystal size difference under two methods 